\title{
ERROR ESTIMATE FOR OPTIMALITY OF DISTRIBUTED PARAMETER CONTROL PROBLEMS VIA DUALITY
}

\author{
W.L. CHAN \\ The Chinese University of Hong Kong \\ Department of Mathematics \\ Hong Kong \\ S.P. YUNG \\ The University of Hong Kong \\ Department of Mathematics \\ Hong Kong
}

(Received April, 1994; Revised February, 1995)

\begin{abstract}
Sharp error estimates for optimality are established for a class of distributed parameter control problems that include elliptic, parabolic, hyperbolic systems with impulsive control and boundary control. The estimates are obtained by constructing manageable dual problems via the extremum principle.
\end{abstract}

Key words: Distributed Parameter Systems, Optimal Control, Duality.

AMS (MOS) subject classifications:49N15, 49N25, 49K20.

\section{Introduction, Notation and Definitions}

We develop a method that generates computable error estimates for optimality as well as dual problems for the optimal control of distributed systems described in the classic work of J.L. Lions [4] and [5]. The approach that we use relies on establishing manageable dual problems as opposed to formidable convex conjugate dual problems. Our approach is elementary in that the quadratic nature of the cost functionals is exploited. The resulting cost functional of the dual system is more explicit than those given in [4] and [5]. Furthermore, we can bypass the requirement of the system equation to be an isomorphism when a conjugate function method is taken in developing a duality theory and our framework permits constrained control sets. The basic idea of our approach is the extremum-principle, which is developed in finite dimensional space in [8] and [1], and here, we successfully extend it to infinite-dimensional problems. Other duality studies for distributed systems can be found in $[2,3,6,7]$.

In the following three sections, we treat error estimates and duality theorems on systems governed by elliptic, parabolic, and hyperbolic equations, respectively. Illustrative examples are given including those involving impulsive control and boundary control. In fact, we believe that the method developed here can cope with almost all situations studied in [4].

We begin with real Hilbert spaces $V, H, \mathcal{U}$ and $\mathcal{H}$. Assume that the injection $V \subseteq H$ is continuous and $V$ is dense in $H$. Identify $H$ with its dual, denote by $V^{\prime}$ the dual of $V$ and we may write 


$$
V \subset H \subset V^{\prime} \text {. }
$$

Both the dual pairing of $V$ and $V^{\prime}$ as well as other Hilbert spaces and their duals are denoted by $\langle\cdot, \cdot\rangle$. We write $(\cdot, \cdot)_{V}$ for the inner product of the Hilbert space $V,\|x\|_{V}$ for $(x, x)_{V}^{\frac{1}{2}}$ and drop $V$ when it is clear from the context which space we are referring to. Inner products and norms of other Hilbert spaces are denoted similarly. We denote by $\mathcal{L}(X, Y)$ the space of continuous linear mappings between the topological vector spaces $X$ and $Y$.

\section{Elliptic Systems}

Assume that we are given operators $B \in \mathcal{L}\left(\mathcal{U}, V^{\prime}\right)$ and $A \in \mathcal{L}\left(V, V^{\prime}\right)$ such that the bilinear form $(A u, v)$ on $V$ is coercive.

For a given $f \in V^{\prime}$ and a control $u \in \mathcal{U}$, we are interested in the system given by

$$
A y=f+B u, \quad y \in V
$$

with state $y:=y(u)$. We are also given an observation equation

$$
z(u)=C y(u)
$$

and a cost functional

$$
J(u, y)=(C y(u), C y(u))_{\mathcal{J C}_{6}}+(N u, u)_{\varkappa}
$$

where $C \in \mathcal{L}(V, \mathfrak{H})$ and $N$ is a Hermitian positive definite operator on $\mathcal{U}$.

Let $\mathcal{u}_{a d}$ (the set of admissible controls) be a given closed convex subset of $\mathcal{U}$. We are interested in the optimal control problem of finding $u_{0}$ and $y_{0}:=y\left(u_{0}\right)$ such that they satisfy $(2.1)$ and

$$
J\left(u_{0}, y_{0}\right)=\inf \left\{J(u, y): u \in \mathcal{U}_{a d}\right\} .
$$

We shall develop a dual problem for this optimal control problem and use it to obtain error estimates for optimality. We now introduce a dual problem.

Let $\Lambda_{\mathfrak{H}}$ be the canonical isomorphism of $\mathfrak{H} \mathscr{C}$ onto its dual $\mathfrak{J C}^{\prime}, C^{*} \in \mathcal{L}\left(\mathfrak{J G}^{\prime}, V^{\prime}\right)$ be the adjoint of $C$. Then for $\psi, \phi \in V$ we have

$$
\left\langle C^{*} \Lambda_{\mathcal{J C}_{6}} C \phi, \psi\right\rangle=\left\langle\Lambda_{\mathcal{J C}_{6}} C \phi, C \psi\right\rangle=(C \phi, C \psi)_{\mathfrak{J}} .
$$

Let $\Lambda_{\mathcal{U}}$ be the canonical isomorphism of $\mathcal{U}$ into its dual $\mathcal{U}^{\prime}, B^{*} \in \mathcal{L}\left(V, \mathcal{U}^{\prime}\right)$ be the adjoint of $B$. Then for $u \in \mathcal{U}, \psi \in V$ we have

$$
\langle B u, \psi\rangle=\left\langle B^{*} \psi, u\right\rangle=\left(\Lambda_{u}^{-1} B^{*} \psi, u\right)_{u} .
$$

Let $A^{*} \in \mathcal{L}\left(V, V^{\prime}\right)$ be the adjoint of $A$. The state $\tilde{p}:=\widetilde{p}(\widetilde{y}) \in V$ of the dual system is defined and given by

$$
A^{*} \tilde{p}=C^{*} \Lambda_{\mathcal{H}_{6}} C \tilde{y}, \quad \tilde{y} \in V .
$$

For $\tilde{y} \in V$ and $\tilde{p}$ given by (2.7), we are interested in those $u \in \mathcal{U}_{a d}$, satisfying

$$
\left(\Lambda_{\mathcal{u}}^{-1} B^{*} \tilde{p}+N u, w-u\right)_{\mathcal{u}} \geq 0 \text { for all } w \in \mathcal{u}_{a d} \text {. }
$$

This restriction on $u$ shall form a characterization for the optimal control. Please see Theorem 2.2 for details. The dual cost functional is

$$
\widetilde{J}(v, \tilde{y})=-(C \tilde{y}, C \tilde{y})_{\mathcal{H}}-(N v, v)_{\mathcal{U}}+2\langle f, \tilde{p}\rangle+\inf _{w \in \mathcal{U}_{a d}} 2\left(\Lambda_{\mathcal{u}^{-1} B^{*} \tilde{p}}+N v, w\right)_{\mathcal{U}} \cdot
$$


The dual problem is to find $v_{0}, \widetilde{y}_{0}$ such that (2.7) holds and

$$
\widetilde{J}\left(v_{0}, \tilde{y}_{0}\right)=\sup \left\{\tilde{J}(v, \tilde{y}: \tilde{y}) \in V, v \in \mathcal{u}_{a d}\right\} .
$$

We have the following lemma regarding the difference between the primal and dual objective functions $J$ and $\widetilde{J}$.

Lemma 2.1: Suppose that $u, v \in \mathcal{U}_{a d}, y$ satisfies (1.1), $\tilde{y} \in V$ and $\tilde{p}$ is given by (2.7). Then

$$
\begin{gathered}
J(u, y)-\tilde{J}(v, \tilde{y})=(C(y-\tilde{y}), C(y-\tilde{y}))+(N(u-v), u-v),+2\left(\Lambda_{\mathcal{u}}^{-1} B^{*} \tilde{p}+N v, u\right) \\
-\inf _{w \in \mathcal{U}_{a d}} 2\left(\Lambda_{u^{-1}} B^{*} \tilde{p}+N v, w\right) .
\end{gathered}
$$

Proof: It follows immediately from the definition of $J$ and $\tilde{J}$ that $J(u, y)-\tilde{J}(v, \tilde{y})$ is the sum of

and twice

But

$$
(C(y-\tilde{y}), C(y-\tilde{y}))+(N(u-v), u-v)
$$

$$
(C \tilde{y}, C y)+(N v, u)-\langle f, \tilde{p}\rangle-\inf _{w \in \mathcal{u}_{a d}}\left(\Lambda_{\tilde{u}}^{-1} B^{*} \tilde{p}+N v, w\right)
$$

$$
(C \tilde{y}, C y)=\left\langle C^{*} \Lambda C \tilde{y}, y\right\rangle=\left\langle A^{*} \tilde{p}, y\right\rangle=\langle A y, \tilde{p}\rangle=\langle f+B u, \tilde{p}\rangle=\langle f, \tilde{p}\rangle+\left(\Lambda_{u^{-1}}^{B^{*}} \tilde{p}, u\right) .
$$

In the above, we have used respectively (2.5), (2.7), (2.1) and (2.6). Now substitute it into (2.12) and we are done.

Theorem 2.2: Suppose that there is $\nu>0$ such that for $u \in \mathcal{U}$,

$$
(N u, u)_{\varkappa} \geqq \nu(u, u)_{\varkappa} \cdot
$$

(i) For all $u, v \in \mathcal{U}_{a d}$, all $y$ satisfying (2.1), all $\tilde{y} \in V$ and all dual state $\tilde{p}$ given by (2.7), we have

$$
\widetilde{J}(v, \tilde{y}) \leqq J(u, y)
$$

(ii) Let $u_{0}$ and $y_{0}$ be the unique solution of (2.1), (2.7) and (2.8) with $y=\tilde{y}$, then $u_{0}$ is the optimal control for the cost functional $J$ of the elliptic system. Furthermore, $J\left(u_{0}, y_{0}\right)=\widetilde{J}\left(u_{0}, y_{0}\right)$, i.e.,

$$
\sup \tilde{J}(v, \tilde{y})=\inf J(u, y)=J\left(u_{0}, y_{0}\right)
$$

where the supremum is taken over $\tilde{y} \in V$ and $v \in \mathcal{U}_{a d}$ and the infimum is over $u \in \mathcal{U}_{a d}$ and $y \in V$ satisfying (2.1).

Proof: From Lemma 2.1, we see that

$$
J(u, y)-\tilde{J}(v, \tilde{y}) \geqq 0 .
$$

The statement about the optimal control $u_{0}$ is just Theorem 1.4 of [4], p. 49. To show that $J\left(u_{0}, y_{0}\right)=\widetilde{J}\left(u_{0}, y_{0}\right)$, we have $y_{0}=y\left(u_{0}\right), \tilde{p}_{0}=\tilde{p}\left(y_{0}\right)$. We have

$$
J\left(u_{0}, y_{0}\right)=J\left(u_{0}, y_{0}\right)-2\left(\Lambda_{u}^{-1} B^{*} \tilde{p}_{0}, u_{0}\right)+2\left(\Lambda_{u^{-1}}^{-1} B^{*} \tilde{p}_{0}, u_{0}\right) .
$$

From (2.13),

$$
J\left(u_{0}, y_{0}\right)=\left(C y_{0}, C y_{0}\right)+\left(N u_{0}, u_{0}\right)
$$

and from the proof of Lemma 2.1, 




Substituting into (2.16) we get

$$
J\left(u_{0}, y_{0}\right)=-\left(C y_{0}, C y_{0}\right)-\left(N u_{0}, u_{0}\right)+2\left\langle f, p_{0}\right\rangle+2\left(\Lambda_{u^{-1}} B^{*} \tilde{p}_{0}+N u_{0}, u_{0}\right) .
$$

But at optimal control $u_{0}$, the variational inequality (2.8) is equivalent to (cf: [4], p. 49 (1.31))

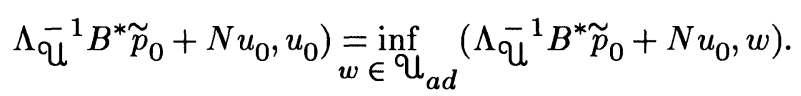

Hence we get

which yields (2.15).

$$
J\left(u_{0}, y_{0}\right)=\tilde{J}\left(u_{0}, y_{0}\right)
$$

The error estimates for optimality are as follows.

Theorem 2.3: Let $u_{0}$ be the optimal control for the cost functional $J$ given by (2.3) under constraints determined by the elliptic system (2.1) and suppose that (2.13) holds. For any $u \in \mathcal{U}_{a d}, y=y(u), \tilde{p}=\tilde{p}(y)$ and $y_{0}=y\left(u_{0}\right)$, we have the following error estimates



and

$$
\left\|C\left(y_{0}-y\right)\right\|^{2}+\left(N\left(u_{0}-u\right), u_{0}-u\right)+2\left(\Lambda_{u}^{-1} B^{*} \tilde{p}+N u, u_{0}\right) \leqq 2\left(\Lambda_{\mathcal{u}}^{-1} B^{*} \tilde{p}+N u, u\right) .
$$

Furthermore, both estimates are sharp.

Proof: From Theorem 2.2,

and so by Lemma 2.1,

$$
J\left(U_{0}, y_{0}\right) \geqq \tilde{J}(v, y)
$$

$$
\begin{gathered}
J(u, y)-J\left(u_{0}, y_{0}\right) \leqq J(u, y)-\tilde{J}(v, y) \\
=(N(u-v), u-v)+2\left(\Lambda_{\mathcal{u}}^{-1} B^{*} \tilde{p}+N v, u\right)-\inf _{w \in \bigcup_{a d}} 2\left(\Lambda_{\mathcal{u}}^{-1} B^{*} \tilde{p}+N v, w\right) .
\end{gathered}
$$

Thus, if we put $v$ equals to $u$ we get (2.17).

To prove (2.18), we apply Lemma 2.1 to both sides of the following inequality

We get

$$
J\left(u_{0}, y_{0}\right)-\tilde{J}(v, y) \leqq J(u, y)-\widetilde{J}(v, y) .
$$

$$
\begin{gathered}
\| C\left(y_{0}-y \|^{2}+\left(N\left(u_{0}-v\right), u_{0}-v\right)+2\left(\Lambda_{\left.u^{-1} B^{*} \tilde{p}+N v, u_{0}\right)}\right.\right. \\
\leqq(N(u-v), u-v)+2\left(\Lambda_{\left.u^{-1} B^{*} \tilde{p}+N v, u\right) .}\right.
\end{gathered}
$$

Putting $v=u$ completes the proof.

Example 2.4: Let $\Omega$ be a bounded open set in $\mathbb{R}^{n}$ such that its closure $\bar{\Omega}$ is a compact manifold with boundary $\Gamma$, which is an $(n-1)$-dimensional smooth manifold. The Euclidean norm of $\mathbb{R}^{n}$ is denoted by $|\cdot|$ and the inner product in $\mathbb{R}^{n}$ is denoted by ordinary multiplication for brevity. Set $V=H_{0}^{1}(\Omega), V^{\prime}=H^{-1}(\Omega), H=L^{2}(\Omega)$. Let $A$ be the second order elliptic operator

$$
A \psi=-\sum_{i, j=1}^{n} \frac{\partial}{\partial x_{i}}\left(a_{i j} \frac{\partial}{\partial x_{j}} \psi\right)+a_{0} \psi
$$


for $\psi \in H_{0}^{1}(\Omega)$ such that

and almost everywhere in $\Omega$,

$$
a_{i j}, a_{0} \in L^{\infty}(\Omega)
$$

and

$$
\sum_{i, j=1}^{n} a_{i j}(x) \xi_{i} \xi_{j} \geqq \alpha\left(\xi_{1}^{2}+\ldots+\xi_{n}^{2}\right), \quad \alpha>0
$$

$$
a_{0}(x) \geqq \alpha
$$

Let the space of controls $U$ be $L^{2}(\Omega)$. Define the set of admissible controls by

$$
\mathcal{U}_{a d}=\{u \in \mathcal{U}: u \geqq 0 \text { almost everywhere in } \Omega\} .
$$

Let $\mathcal{H}_{6}=H$ and let $C$ be the injection of $V$ into $H$. Take $\Lambda_{\mathcal{\Psi}}$ and $B$ be the identity operator on $L^{2}(\Omega)$, and let $f \in H^{-1}(\Omega)$ and $N$ be a positive definite Hermitian operator on $L^{2}(\Omega)$ satisfying (2.13). If the primal problem is to find the optimal control minimizing cost

under the constraints

$$
J(u, y)=\int_{\Omega}|y(x)|^{2} d x+\int_{\Omega}(N v)(x) v(x) d x
$$

$$
\left\{\begin{array}{cc}
A y=f+u & \text { in } \Omega \\
y=0 & \text { on } \Gamma \\
u \geqq 0 & \text { almost everywhere in } \Omega
\end{array}\right.
$$

then the dual problem is to maximize the cost function

$$
\widetilde{J}(v, \tilde{y})=-\int_{\Omega}|\tilde{y}(x)|^{2} d x-\int_{\Omega}(N v)(x) v(x) d x+2\langle f, \tilde{p}\rangle+2 \inf _{w \in \cup_{a d}} \int_{\Omega}(\tilde{p}+N v)(x) w(x) d x
$$

under the constraints

$$
\left\{\begin{array}{cc}
A^{*} \tilde{p}=\tilde{y} & \text { in } \Omega \\
\tilde{p}=0 & \text { on } \Gamma .
\end{array}\right.
$$

Furthermore, the error estimates of Theorem 2.3 hold for this problem.

\section{Parabolic Systems}

We continue to use the notation of Section 2 and introduce additional notation.

If $V$ is a Hilbert space, we write $L^{2}(0, T ; V)$ for the space (of equivalence classes) of functions defined on the open interval $] 0, T[$ with values in $V$ such that

We define

$$
\int_{0}^{T}\|f(t)\|^{2} d s<\infty .
$$

$$
W(0, T)=\left\{f: f \in L^{2}(0, T ; V), \frac{d f}{d t} \in L^{2}\left(0, T ; V^{\prime}\right)\right\}
$$

Suppose that we are given a family of operators $A(t) \in \mathcal{L}\left(L^{2}(0, T ; V), L^{2}\left(0, T ; V^{\prime}\right)\right)$ such that for $\psi, \phi \in V$, the function

$$
t \mapsto(A(t) \psi, \phi)
$$


is continuously differentiable in $[0, T]$,

$$
|(A(t) \psi, \phi)| \leqq c\|\psi\|\|\phi\|
$$

and there exists a $\lambda$ such that for $\phi \in V, 0<t<T$,

$$
(A(t) \phi, \phi)+\lambda\|\phi\|_{H}^{2} \geqq \alpha\|\phi\|_{V}^{2}, \alpha>0 .
$$

Assume that the cost functional is given by

$$
J(u, y)=\|C y(u)\|_{\mathfrak{J G}}^{2}+(N u, u)_{\mathcal{U}}
$$

where $y(u) \in W(0, T)$, the observation operator $C \in \mathcal{L}(W(0, T), \mathcal{H})$, and $N \in \mathcal{L}(\mathcal{U}, \mathcal{U})$ with

$$
(N u, u)_{น} \geqq \nu\|u\|_{\stackrel{2}{u}, \quad \nu>0 .}
$$

Let $B \in \mathcal{L}\left(\mathcal{U}, L^{2}\left(0, T ; V^{\prime}\right)\right), f \in L^{2}\left(0, T ; V^{\prime}\right)$ and $y_{0} \in H$ be given. We study (cf: [4], p. 114, Theorem 2.1) the problem of minimizing cost $J$ over a parabolic system given by

$$
\left\{\begin{array}{c}
\frac{d}{d t} y+A(t) y=f+B u \\
y(0)=y_{0}
\end{array}\right.
$$

For $u$ in the set $\mathcal{U}_{a d}$ of admissible controls and $y \in L^{2}(0, T ; V)$.

Let the dual system by given by

$$
\left\{\begin{array}{c}
-\frac{d}{d t} \tilde{p}+A^{*}(t) \tilde{p}=C^{*} \Lambda_{{ }_{\zeta}} C \tilde{y} \\
\tilde{p}(T)=0
\end{array}\right.
$$

where $\tilde{p} \in L^{2}(0, T ; V)$. We shall be interested in those $u \in \mathcal{U}_{a d}$ satisfying

$$
\left(\Lambda_{\mathcal{u}}^{-1} B^{*} \tilde{p}+N u, w-u\right)_{u} \geqq 0, \text { for all } w \in \mathcal{U}_{a d}
$$

with $\tilde{p}$ satisfying (3.6). We define the dual cost functional by

$\widetilde{J}(v, \tilde{y})=-\|C \tilde{y}(v)\|_{{ }^{\mathcal{G}}}^{2}-(N v, v)_{\mathcal{U}}+2\langle f, \tilde{p}\rangle+2(y(0), \tilde{p}(0))+\inf _{w \in \mathcal{U}_{a d}} 2\left(\Lambda_{\mathcal{u}}^{-1} B^{*} \tilde{p}+N v, w\right)$.

The dual problem is to maximize $\widetilde{J}(v, \tilde{y})$ subject to $\tilde{y} \in L^{2}(0, T, V), \tilde{p}$ satisfying (3.6) and $v \in \mathcal{u}_{a d}$.

Lemma 3.1: If $u, v \in \mathcal{U}, y(u)$ satisfies (3.5), $\tilde{y} \in V$ and $\tilde{p}$ satisfies (3.6) then

$$
\begin{gathered}
J(u, y)-\tilde{J}(v, \tilde{y})=\|C(y-\tilde{y})\|_{\mathfrak{J G}^{2}}^{2}+(N(u-v), u-v)+2\left(\Lambda_{\mathcal{u}}^{-1} B^{*} \tilde{p}+N \nu, u\right) \\
-\inf _{w \in \mathcal{U}_{a d}} 2\left(\Lambda_{\mathcal{u}^{-1}} B^{*} \tilde{p}+N v, w\right) .
\end{gathered}
$$

Proof: One can verify this lemma directly as before. Alternatively, we can estimates $J(u, v)$ $-\widetilde{J}(v, \widetilde{y})$ from below and see how $\widetilde{J}(v, \widetilde{y})$ drops out. First of all, for any symmetric bilinear form we have an identity 


$$
(a, a)+(b, b)=2(b, a)+(a-b, a-b) .
$$

Applying the identity to the bilinear forms $(\cdot, \cdot)_{\mathcal{J G}}$ and $u, v \mapsto(N u, v)_{\mathcal{U}}$, we see that $J(u, y)$ $-\widetilde{J}(v, \tilde{y})$ equals

$$
\begin{gathered}
\|C(y-\tilde{y})\|^{2}+(N(u-v), u-v)+2[(C \tilde{y}, C y)+(N v, u) \\
\left.-\langle f, \tilde{p}\rangle-(y(0), \tilde{p}(0)) \operatorname{-inf}_{w \in \bigcup_{a d}}\left(\Lambda_{u}^{-1} B^{*} \tilde{p}+N v, w\right)\right] .
\end{gathered}
$$

On the other hand, we have

So by $(3.6)$,

$$
(C \tilde{y}, C y)=\left\langle C^{*} \Lambda_{\mathcal{H}} C \tilde{y}, y\right\rangle
$$

$$
\begin{aligned}
& (C \tilde{y}, C y)=\int_{0}^{T}\left(-\frac{d}{d t} \tilde{p}+A^{*}(t) \tilde{p}, y\right) d t \\
& =\int_{0}^{T}\left(\frac{d}{d t} y+A(t) y, \tilde{p}\right) d t+(y(0), \tilde{p}(0)),
\end{aligned}
$$

where we have used Green's formula and

$$
\int_{0}^{T}\left(\frac{d}{d t} y, \widetilde{p}\right) d t+\int_{0}^{T}\left(\frac{d}{d t} \widetilde{p}, y\right) d t=(y(T), \widetilde{p}(T))-(y(0), \widetilde{p}(0)) .
$$

Using (3.5) we get

$$
(C \tilde{y}, C y)=\langle f, \tilde{p}\rangle+(B u, \tilde{p})+(y(0), \tilde{p}(0)) .
$$

Substituting this into (3.10), we get

and the proof is now complete.



Theorem 3.2: Assume that (3.1), (3.2), (3.3) hold. Then

(i) The cost functional $J(u, y)$ of the system (3.5) and the dual cost functional $\tilde{J}(v, \tilde{y})$ of the system (3.6) satisfy

$$
\widetilde{J}(v, \tilde{y}) \leqq J(u, y)
$$

for all $u, v \in \mathrm{U}_{a d}$, all $y$ satisfying (3.5), all $\tilde{y} \in V$, and all dual state $\tilde{p}$ given by (3.6).

(ii) The optimal control $u_{0}$ and the corresponding $y_{0}$ are characterized by (3.5),(3.6), (3.7) with $\tilde{y}$ taken to be $y$. Furthermore, we have

$$
\sup \tilde{J}(v, \tilde{y})=\inf J(u, y)=J\left(u_{0}, y_{0}\right)
$$

where the infimum is taken over all $u \in \mathcal{U}_{\text {ad }}$ and $y$ satisfying (3.5), and the supremum is over all $(\tilde{y}, \tilde{p})$ satisfying $(3.6)$ and $v \in \mathcal{U}_{a d}$.

(iii) If we put $y_{0}:=y\left(u_{0}\right), \widetilde{p}:=\widetilde{p}(y)$ and take $u$ in $\mathcal{u}_{a d}$, $v$ in $\mathcal{u}_{a d}(\tilde{p})$, then we have the following error estimates:

$$
\begin{gathered}
\left\|C\left(y-y_{0}\right)\right\|^{2}+\left(N\left(u_{0}-v\right), u_{0}-v\right)+2\left(\Lambda_{u^{-1}} B^{*} \tilde{p}+N v, u_{0}-v\right) \\
\leqq(N(u-v), u-v)+2\left(\Lambda_{u^{-1}} B^{*} \tilde{p}+N v, u-v\right),
\end{gathered}
$$

and 


$$
J(u, y)-J\left(u_{0}, y_{0}\right) \leqq(N(u-v), u-v)+2\left(\Lambda_{u^{-1}} B^{*} \tilde{p}+N v, u\right)-2 \inf _{w \in \mathcal{U}_{a d}}\left(\Lambda_{u^{-1}} B^{*} \tilde{p}+N v, w\right) .
$$

Proof: Same as corresponding theorems in Section 2. The characterization of the optimal control is in [4], p. 114, Theorem 2.1.

Example 3.3: Take $\Omega$ as in Example 1.4. Put $Q=\Omega \times] 0, T[, \Sigma=\Gamma \times] 0, T\left[\right.$. Let $a_{i j}$ be functions in $Q$ such that

$$
\left\{\begin{array}{l}
a_{i j} \in L^{\infty}(Q) \\
\sum_{i, j=1}^{n} a_{i j}(x, t) \xi_{i} \xi_{j} \geqq \alpha\left(\sum_{i=1}^{n} \xi_{i}^{2}\right), \quad \alpha>0, \quad \xi_{i} \in \mathbb{R} .
\end{array}\right.
$$

For $\psi \in H_{0}^{1}(\Omega)$, define $A(t)$ by

$$
A(t) \psi=-\sum_{i, j=1}^{n} \frac{\partial}{\partial x_{i}}\left(a_{i j}(x, t) \frac{\partial \psi}{\partial x_{j}}\right) .
$$

Let $V \in H_{0}^{1}(\Omega), H=L^{2}(\Omega), \mathcal{U}=\mathcal{U}^{\prime}=L^{2}(Q), \mathfrak{H} \mathcal{J}^{\prime}=\mathfrak{J}^{\prime}=L^{2}(Q)$. Also take $B$ and $\Lambda_{\mathcal{U}}$ to be the identity mapping, $C: L^{2}(0, T ; V) \rightarrow L^{2}(Q)$ be the injection map and let

$$
\mathcal{U}_{a d}=\{u \in \mathcal{U}: u \geqq 0 \text { almost everywhere in } Q\} .
$$

For $f \in L^{2}\left(0, T ; H^{-1}(\Omega)\right)$, we consider a mixed Dirichlet problem for a second order parabolic equation:

$$
\left\{\begin{array}{cc}
\frac{d}{d t} y+A(t) y=f+u & \text { in } Q \\
\left.y\right|_{\Sigma}=0 & \\
y(x, 0)=y_{0}(x) & x \in \Omega \\
y \in L^{2}\left(0, T ; H_{0}^{1}(\Omega)\right) . &
\end{array}\right.
$$

The optimal control problem is to minimize the cost given by

$$
J(u, y)=\int_{Q}\left(|y(x, t)|^{2}+(N u)(x, t) u(x, t)\right) d x d t .
$$

We define the dual problem to be maximizing the dual cost functional

$$
\begin{gathered}
\tilde{J}(v, \tilde{y})=\int_{Q}\left(-|\tilde{y}|^{2}-(N v) v\right) d x d t+2\langle f, \tilde{p}\rangle+2 \int_{\Omega} y(0, x) \tilde{p}(0, x) d x \\
+2 \inf _{w \in \mathcal{U}_{a d}} \int_{Q}(\tilde{p}+N v) w d x d t
\end{gathered}
$$

over the adjoint system governed by

$$
\left\{\begin{array}{cc}
\frac{d}{d t} \tilde{p}+A^{*}(t) \tilde{p}=\tilde{y} & \text { in } Q \\
p=0 & \text { on } \Sigma \\
p(x, \Gamma)=0 & \\
p \in L^{2}\left(0, T ; H_{0}^{1}(\Omega)\right) . &
\end{array}\right.
$$


The techniques we have used can also be applied to construct the dual problem to the problem of impulsive control of linear evolution problems. We use on in [5] Chapter 2 as an example.

Example 3.4: Suppose that the regularity conditions in [4], p. 182 are imposed on $\Omega$, which is a domain in $\mathbb{R}^{n}$, with $n \leq 3$. Let $A$ be the elliptic operator described in Example 2.4. Unless otherwise specified, we shall continue to use the notation of Example 3.3. Let $b$ be given in $\Omega$. We denote by $\delta(x-b)$ the Dirac mass at the point $b$. Let the cost functional be

$$
J(u, y)=\int_{\Omega}|y(x, T ; u)|^{2} d x+k \int_{0}^{T} \int_{\Omega}|u(x, t)|^{2} d x d t
$$

where $k$ is a given positive real number and $y=y(x, t ; u)$ is the state of the system given by

$$
\left\{\begin{array}{cc}
\frac{d y}{d t}+A y=u(t) \delta(x-b) & \text { in } Q \\
y=0 & \text { on } \Sigma \\
y(x, 0)=0 & \text { in } \Omega .
\end{array}\right.
$$

The space of controls is

$$
\mathcal{U}=\left\{u: u \in L^{2}(0, T), y(\cdot, T ; u) \in L^{2}(\Omega)\right\},
$$

with norm

$$
\|u\|_{\mathrm{u}}=\left(\int_{0}^{T}|u|^{2} d t+\int_{\Omega}|y(x, T ; u)|^{2} d x\right)^{1 / 2}
$$

and we assume that the admissible controls $\mathcal{U}_{a d}$ is a closed convex non-empty subset of $\mathcal{U}$.

Let the dual state $\tilde{p}$ be the solution of the backward system

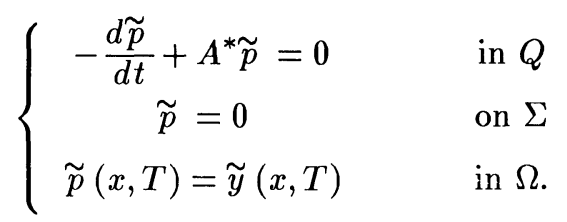

By defining the dual cost functional to be

$$
\begin{gathered}
\tilde{J}(v, \tilde{y})=-\int_{\Omega}|\tilde{y}(x, T ; v)|^{2} d x-k \int_{0}^{T}|v(t)|^{2} d t+2 \int_{0}^{T} v(t) \tilde{p}(b, t) d t \\
\quad+2 \inf _{w \in \cup_{a d}} \int_{0}^{T}(\tilde{p}(b, t)+k v) w d t
\end{gathered}
$$

we may conclude, through proving parallel results of Theorem 3.1 and Theorem 3.2 , and the problem of maximizing $\widetilde{J}$ with $\widetilde{y}(x, T)$ and $\widetilde{p}$ given by (3.19), is a dual problem to that of minimizing $J$ with $u \in \mathcal{U}_{a d}$ for the system (3.17). Corresponding error estimates may be established similarly.

\section{Hyperbolic Systems}

We continue to use the notation of Section 2. Consider a family of operators $A(t) \in \mathcal{L}\left(V, V^{\prime}\right)$ satisfying conditions (3.1), (3.2) and for all $\psi, \phi \in V$ 


$$
(A(t) \psi, \phi)=(A(t) \phi, \psi) .
$$

Suppose $B \in \mathcal{L}\left(\mathcal{U}, L^{2}(0, T ; H)\right), f \in L^{2}(0, T, H), y_{0} \in V, y_{1} \in H$ and $y \in L^{2}(0, T ; V)$ such that $\frac{d y}{d t} \in L^{2}(0, T ; H)$. The system that we are interested in is

$$
\left\{\begin{array}{c}
\frac{d^{2}}{d t^{2}} y+A(t) y=f+B v \\
y(0)=y_{0} \\
\frac{d y}{d t}(0)=y_{1} .
\end{array}\right.
$$

The observation operator is $C \in \mathcal{L}\left(L^{2}(0, T ; H) ; \mathfrak{H}\right)$ and the cost functional is

$$
J(u, y)=\|C y(u)\|_{\mathfrak{J} 6}^{2}+(N u, u)_{\mathcal{U}}
$$

where $N$ is as in Section 2 satisfying (2.13). The problem is to minimize $J(u, y)$ for $u \in \mathcal{U}_{a d}$.

For $\tilde{y} \in L^{2}(0, T ; H), \tilde{p} \in L^{2}(0, T ; V)$ and $\frac{d \widetilde{p}}{d t} \in L^{2}(0, T ; H)$, we define the dual system

and the cost functional by

$$
\left\{\begin{aligned}
\frac{d^{2}}{d t^{2}} \tilde{p}+A(t) \tilde{p} & =C^{*} \Lambda_{\mathcal{H}} C \tilde{y} \\
\tilde{p}(T) & =0 \\
\frac{d}{d t} \tilde{p}(T) & =0
\end{aligned}\right.
$$

$$
\begin{gathered}
\tilde{J}(v, \tilde{y})=-\|C \tilde{y}\|^{2}-(N v, v)+2\langle f+B v, \tilde{p}\rangle-2\left(y(0), \frac{d}{d t} \tilde{p}(0)\right)+2\left(\tilde{p}(0), \frac{d}{d t} y(0)\right) \\
+\inf _{w \in \mathcal{U}_{a d}} 2\left(\Lambda_{\tilde{u}}^{-1} B^{*} \tilde{p}+N v, w\right) .
\end{gathered}
$$

The proofs of the following lemma and theorem are the same as those in Section 3.

Lemma 4.1: If $y(u)$ satisfies (4.2) and $\widetilde{y}(v)$ satisfies (4.4), then

$$
\begin{aligned}
& J(u, y)-\tilde{J}(v, \tilde{y})=\|C(y-\tilde{y})\|^{2}+(N(u-v), u-v) \\
& +2\left(\Lambda_{\mathcal{u}}^{-1} B^{*} \tilde{p}+N v, u\right) \inf _{w \in \mathcal{u}_{a d}} 2\left(\Lambda_{u^{-1}} B^{*} \tilde{p}+N v, w\right) .
\end{aligned}
$$

Theorem 4.2: Assume that (3.1), (3.2), (3.3) hold. Then

(i) For all $u, v \in \mathcal{U}_{a d}$, all $y$ satisfying $(4.2)$, all $\tilde{y} \in L^{2}(0, T ; H)$ and all dual state $\tilde{p}$ given by (4.4), we have the inequality

$$
\tilde{J}(v, \tilde{y}) \leqq J(u, y)
$$

(ii) The optimal control $u_{0}$ is characterized by (4.2) and (4.4) with $y, \widetilde{p} \in L^{2}(0, T ; V), \frac{\partial y}{\partial x}$, $\frac{\partial \widetilde{p}}{\partial x} \in L^{2}(0, T ; H)$ and $\tilde{y}:=y$ such that

$$
\left(\Lambda_{u}^{-1} B^{*} \tilde{p}+N v, u-v\right)_{u} \geqq 0, \quad \forall u \in \mathcal{U}_{a d} .
$$

(iii) If $u_{0}$ and $y_{0}$ give the optimal control, then

$$
\sup \tilde{J}(v, \tilde{y})=\inf J(u, y)=J\left(u_{0}, y_{0}\right),
$$

where $u, v \in \mathcal{U}_{a d}, y$ satisfies $(4.2)$ and $(\tilde{y}, \tilde{p})$ satisfies (4.4). 
(iv) If we put $y_{0}:=y\left(u_{0}\right), \tilde{p}=\tilde{p}(y)$ and take $u, v \in \mathcal{U}_{a d}$, then we have the following error estimates:

$$
\begin{gathered}
\left\|C\left(y-y_{0}\right)\right\|^{2}+\left(N\left(u_{0}-v\right), u_{0}-v\right)+2\left(\Lambda_{u^{-1}} B^{*} \tilde{p}+N v, u_{0}-v\right) \\
\leqq(N(u-v), u-v)+2\left(\Lambda_{u^{-1}} B^{*} \tilde{p}+N v, u-v\right),
\end{gathered}
$$

and

$J(u, y)-J\left(u_{0}, y_{0}\right) \leqq(N(u-v), u-v)+2\left(\Lambda_{u^{-1}} B^{*} \tilde{p}+N v, u\right)-\underset{w \in \operatorname{unf}_{a d}}{2}\left(\Lambda_{\mathcal{u}^{-1}}^{-1} B^{*} \tilde{p}+N v, w\right)$.

The condition of optimality in (ii) is just Theorem 2.1 of [4], p. 284.

We demonstrate below that a similar approach may be applied to boundary control problems (cf: p. 321 of [4]).

Example 4.3: We use the same notation as Example 3.3 with the requirement that $a_{i j}=a_{j i}$ for all $i, j$. The state $y$ is the solution of the state equation $\left(\nu_{A}\right.$ is the outward normal of $\left.\Omega\right)$

$$
\left\{\begin{array}{cc}
\frac{\partial^{2} y}{\partial t^{2}}+A y=f & \text { in } Q \\
\frac{\partial y}{\partial \nu_{A}}=u & \text { on } \Sigma \\
y(x, 0)=y_{0}(x), & x \in \Omega \\
\frac{\partial y(x, 0)}{\partial t}=y_{1}(x), & x \in \Omega .
\end{array}\right.
$$

Let the primal cost function be

$$
J(u, y)=\int_{\Omega}|y(u)|^{2} d x d t+(N u, u) L^{2}(\Sigma)
$$

such that $N \in \mathcal{L}\left(L^{2}(\Sigma) ; L^{2}(\Sigma)\right)$ satisfies (3.4) for some $\nu>0$. Let $\mathcal{U}_{a d}$ be a closed convex subset of $\mathcal{U}=L^{2}(\Sigma)$. Then there exists an optimal control $u$ in $\mathcal{U}_{a d}$ (see p. 321 of [4]).

Now the dual system given by Theorem 4.2 is to maximize

$$
\begin{gathered}
\tilde{J}(v, \tilde{y})=-\int_{\Omega}|\tilde{y}|^{2} d x d t-(N v, v)+2\langle f, p\rangle+2(v, p)-2 \int_{\Omega} \frac{\partial p}{\partial x}(0, x) y(0, x) d x \\
\quad+2 \int_{\Omega} p(0, x) \frac{\partial y}{\partial x}(0, x) d x+2 \inf _{w \in \mathcal{U}_{a d}}(p+N v, w)
\end{gathered}
$$

subject to

$$
\left\{\begin{aligned}
\frac{\partial^{2} p}{\partial t^{2}}+A p & =f & & \text { in } Q \\
\frac{\partial}{\partial \nu_{A}} p & =0 & & \text { on } \Sigma \\
p(x, T) & =0 & & x \in \Omega \\
\frac{\partial p}{\partial t}(x, T) & =0 & & x \in \Omega .
\end{aligned}\right.
$$

The error estimates can be constructed similarly. 


\section{References}

[1] Arthurs, A.M., Complementary Variational Principles, 2nd Edition, Oxford University, Press, New York 1980.

[2] Barbu, V. and Precupanu, Th., Convexity and Optimization in Banach Spaces, Sijthoff \& Noordhoff, Netherlands 1978.

[3] Chan, W.L. and Ho, L.F., Lower bounds and duality in the optimal control of distributed systems, J. Math. Anal. Appl. 70 (1978), 530-545.

[4] Lions, J.L., Optimal Control of Systems Governed by Partial Differential Equations, Springer-Verlag, Berlin 1971.

[5] Lions, J.L., Function Spaces and Optimal Control of Distributed Systems, Inst. of Math., Federal Univ. of Rio de Janiero 1980.

[6] Mackenroth, U., Weak duality for parabolic boundary control problems, J. Math. Anal. Appl. 90 (1980), 393-407.

[7] Mossino, J., An application of duality to distributed optimal control problems with constraints on the central and the state, J. Math. Anal. Appl. 50 (1975), 334-342.

[8] Noble, B. and Sewell, B., One dual extremum principles in applied mathematics, J. Inst. Maths. and Appl. 9 (1972), 124-193. 


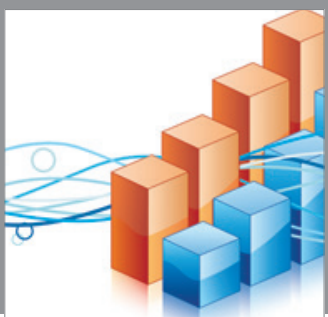

Advances in

Operations Research

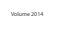

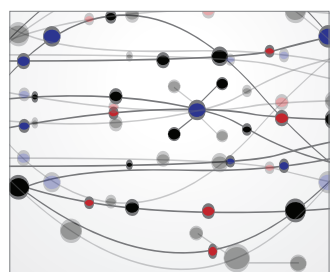

\section{The Scientific} World Journal
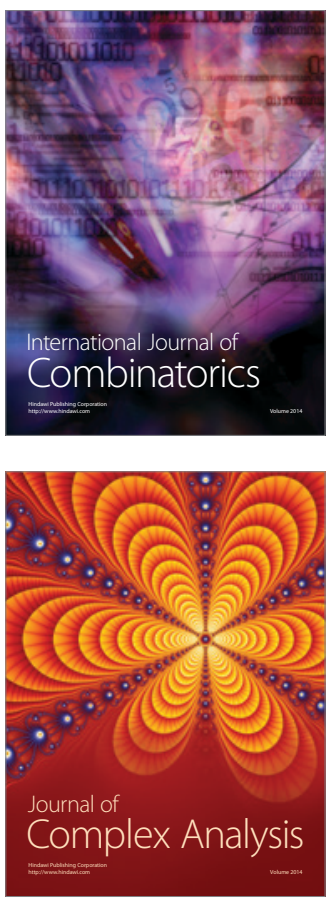

International Journal of

Mathematics and

Mathematical

Sciences
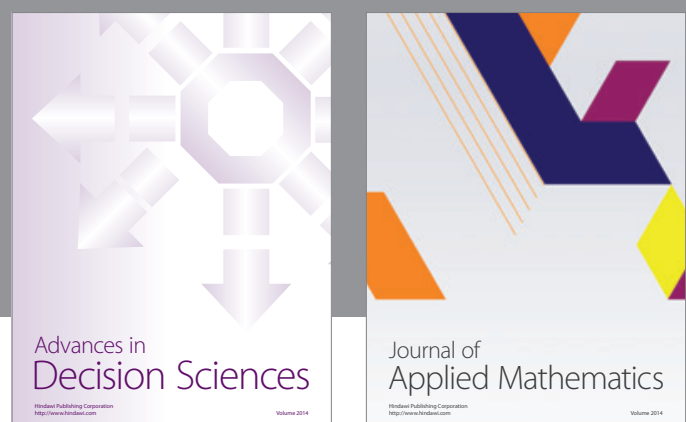

Journal of

Applied Mathematics


Submit your manuscripts at http://www.hindawi.com


Mathematical Problems in Engineering
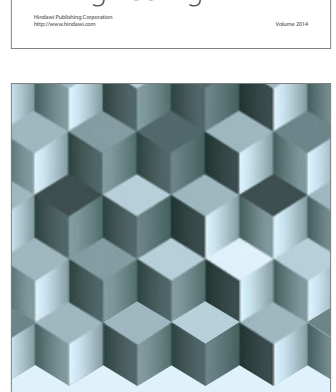

Journal of

Function Spaces
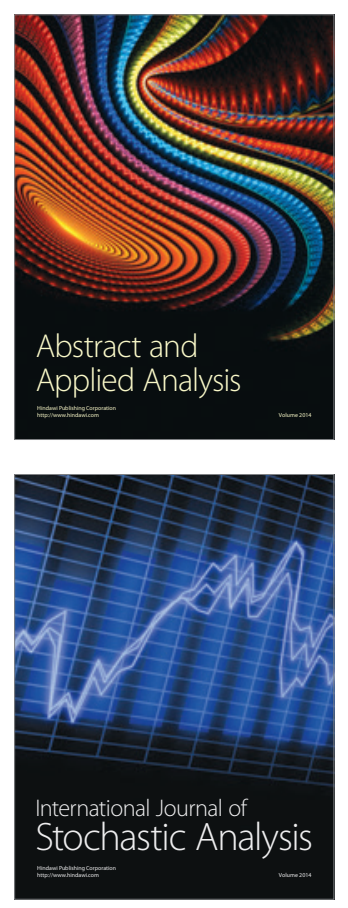



ournal of

Probability and Statistics

Promensencen
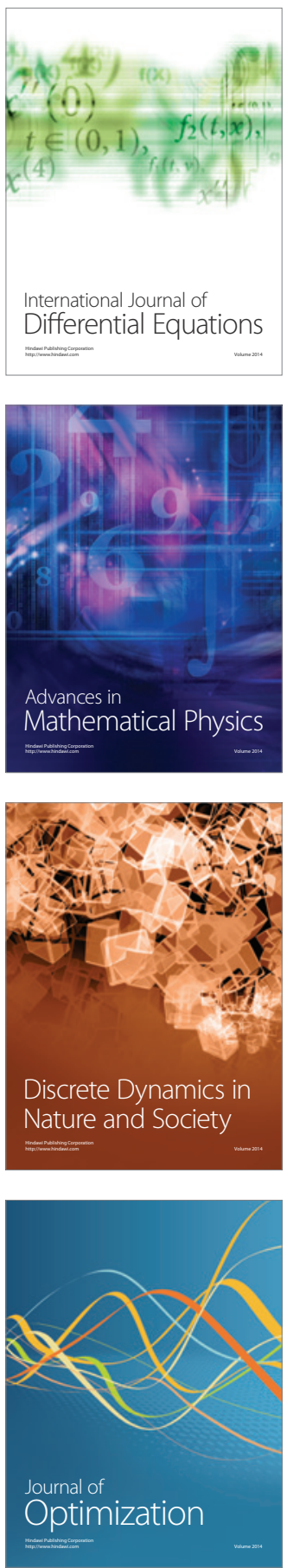